\title{
Optic neuropathy associated with the use of over-the-counter sexual enhancement supplements
}

This article was published in the following Dove Press journal:

Clinical Ophthalmology

29 October 2014

Number of times this article has been viewed

\section{Sapir Z Karli \\ Sophie D Liao \\ Andrew R Carey \\ Byron L Lam \\ Sara T Wester}

Bascom Palmer Eye Institute, University of Miami Miller School of Medicine, Miami, FL, USA
Correspondence: Sara T Wester Bascom Palmer Eye Institute, $900 \mathrm{NW}$

17th Street, Miami, FL 33I36, USA

$\mathrm{Tel}+\mathrm{I} 3053266132$

Email swester2@med.miami.edu
Abstract: This case report details an association of the use of over-the-counter sexual enhancement supplements with atypical optic neuropathy. A 42-year-old man presented with right-sided headache and vision loss of the right eye, which deteriorated to a single quadrant of hand motion over 11 days. Serial orbital magnetic resonance imaging scans demonstrated progressive orbital optic nerve enhancement extending into the optic canal despite high-dose steroid treatment. The patient eventually admitted to using several over-the-counter sexual enhancement supplements prior to the onset of symptoms and throughout the course of his steroid treatment, which he subsequently discontinued. His vision improved to 20/200 with an expanded visual field. Anterior ischemic optic neuropathy has been reported in association with phosphodiesterase (PDE)-5 inhibitor use, but visual loss in association with unregulated sexual enhancement supplements has not been studied. While one case cannot establish association, our case is suggestive of potential dangers of over-the-counter sexual enhancement supplements, which may contain PDE-5 inhibitors, "male hormones," and "substances that enhance blood production." The case also underscores the importance of obtaining a careful history of supplements in patients with optic neuropathies.

Keywords: optic neuropathy, PDE-5 inhibitor, sildenafil

\section{Introduction}

Phosphodiesterase (PDE)-5 inhibitors are commonly prescribed for erectile dysfunction. ${ }^{1-4}$ Inhibition of PDE-5 leads to vasodilation, which may affect ophthalmic circulation and reduce optic nerve perfusion., ${ }^{2,45}$ Ocular complications have been reported to include anterior ischemic optic neuropathy (AION), posterior ischemic optic neuropathy, color perception impairment, optic atrophy, angle closure glaucoma, branch retinal artery occlusion, and central serous chorioretinopathy., ${ }^{2,3,5-7}$

Posterior ischemic optic neuropathy has been reported to have a possible association with PDE inhibitors and Chinese "health products" containing PDE-5 inhibitors. Several reported cases have highlighted the importance of considering this in the differential diagnosis of patients with suspected retrobulbar optic neuritis or a compressive lesion when the clinical course is atypical and refractory to corticosteroid treatment. ${ }^{6,7}$

Initial reports on the link between PDE-5 inhibitors and AION were inconsistent. ${ }^{1,3-5,8}$ The strongest and most recent data demonstrate a small but clinically and statistically significant increase in risk of AION from PDE-5 inhibitor exposure. ${ }^{9}$ Several prospective studies are in progress. ${ }^{10,11}$ Visual loss in association with the use of unregulated sexual enhancement supplements has not been studied. Herein, we describe a case of 
atypical optic neuropathy associated with the use of overthe-counter sexual enhancement supplements.

\section{Case report}

A 42-year-old healthy man was evaluated initially elsewhere for acute right-sided headache followed by visual loss of the right eye with pain on eye movement. Best-corrected visual acuity was 20/400 in the right eye and 20/20 in the left eye, with a right relative afferent pupillary defect. The right eye had $30 \%$ red desaturation and an inferonasal visual field defect. Optic disc edema was not detected, and a brain magnetic resonance imaging (MRI) without contrast was unremarkable. After 5 days of deteriorating vision, the patient was prescribed $40 \mathrm{mg}$ prednisone daily.

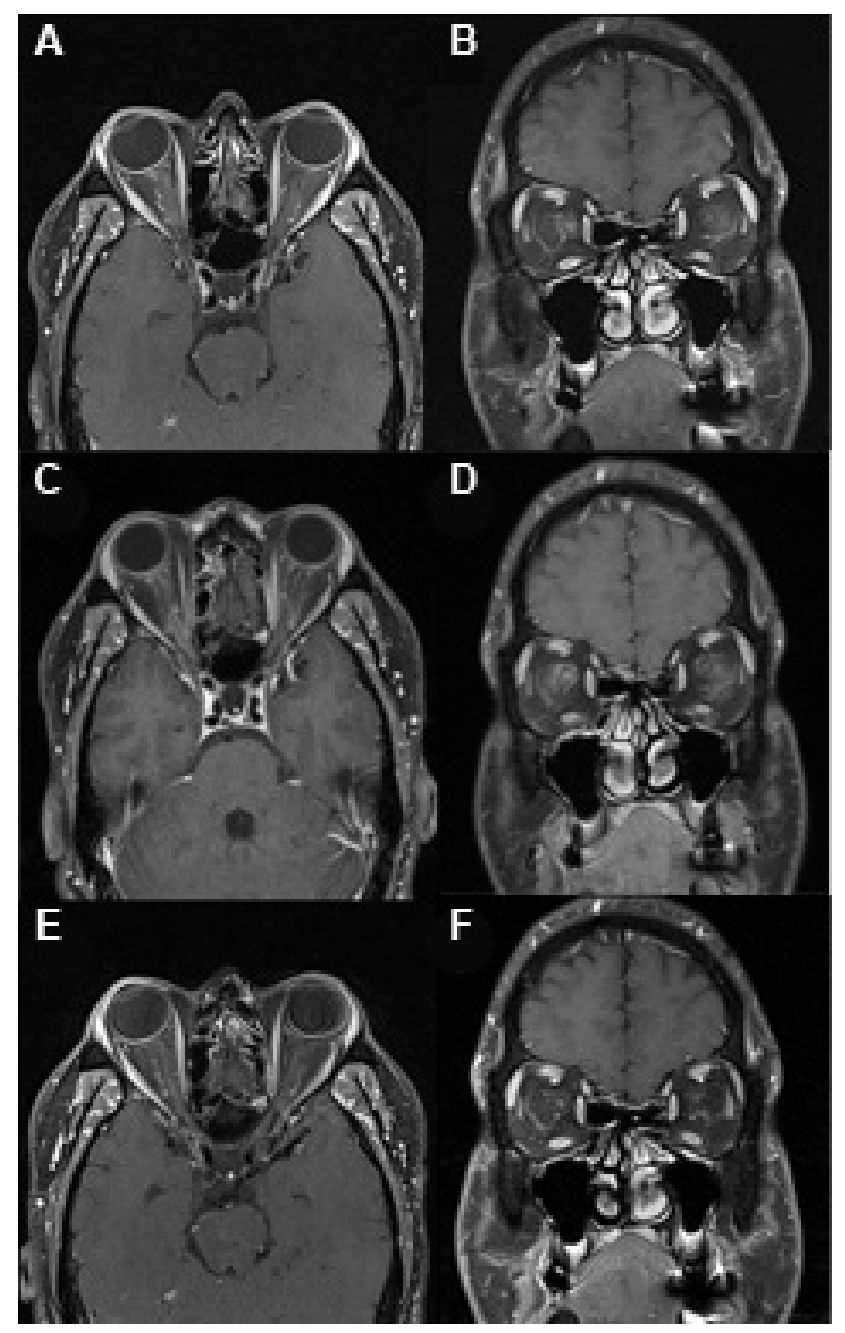

Figure I Orbital magnetic resonance imaging scans.

Notes: (A) Axial and (B) coronal views (postgadolinium TI with fat saturation) on presentation, showing right optic nerve and nerve sheath enhancement. (C) Axial and (D) coronal views 3 weeks after presentation, showing right optic nerve and nerve sheath enhancement extending into the optic canal. (E) Axial and (F) coronal views 7 weeks after presentation, showing decreased right optic nerve and nerve sheath enhancement.
The patient was then referred to our clinic for progressive visual loss and persistent pain with eye movement in the right eye. At initial presentation, 7 days after onset of symptoms, vision in the right eye was hand motion temporally with a relative afferent pupillary defect. The optic nerve had mild edema with blurring of the superior margin. Orbit MRI with contrast revealed enhancement of the right optic nerve and sheath (Figure 1A and B), as well as nonspecific periventricular, deep, and subcortical white matter lesions. The patient was started on intravenous methylprednisolone $1 \mathrm{~g}$ daily, with a plan to assess clinical response frequently, as the MRI optic nerve and sheath enhancement suggested an inflammatory component. ${ }^{12}$

Repeat examination at 11 days after the onset of symptoms demonstrated narrowing of the visual field of the right eye to hand motion in a small temporal island and persistent right afferent pupillary defect. The right optic nerve head was diffusely edematous without hemorrhage (Figure 2A), and the left optic nerve head was normal with a 0.5 cup-to-disc ratio (Figure 2B). A systemic workup for inflammatory etiologies was unremarkable, including anti-aquaporin-4 antibody, anti-neutrophil cytoplasmic antibodies, angiotensin-converting enzyme, rapid plasma reagin, and fluorescent treponemal antibody, as well as Leber hereditary optic neuropathy genotyping, chest $\mathrm{X}$-ray, and cerebral spinal fluid analysis. Due to lack of improvement in vision, intravenous methylprednisolone was discontinued after 8 days and the patient was started on oral prednisone $60 \mathrm{mg}$ daily for 1 week, with a taper of $40 \mathrm{mg}$ daily for 1 week, followed by $20 \mathrm{mg}$ daily for 1 week.

Three weeks after the onset of symptoms, the right eye vision was still hand motion only in the superotemporal quadrant. The right optic nerve head showed persistent edema and the left optic nerve remained normal (Figure 2C and D). Goldmann visual field (GVF) showed severe constriction of the visual field, with a small superotemporal and superonasal island of vision remaining (Figure 3A). Repeat MRI showed enhancement of the right optic nerve extending to the optic canal (Figure 1C and D). Given the progressive clinical course and the lack of significant improvement with intravenous corticosteroids, atypical optic neuritis with perineuritis, ischemic optic neuropathy, and malignant optic glioma were considered. After discussing the case in a multidisciplinary meeting, the decision was made to observe closely and continue prednisone with the aforementioned taper, with the option of an optic nerve or sheath biopsy should the patient's condition worsen further. 

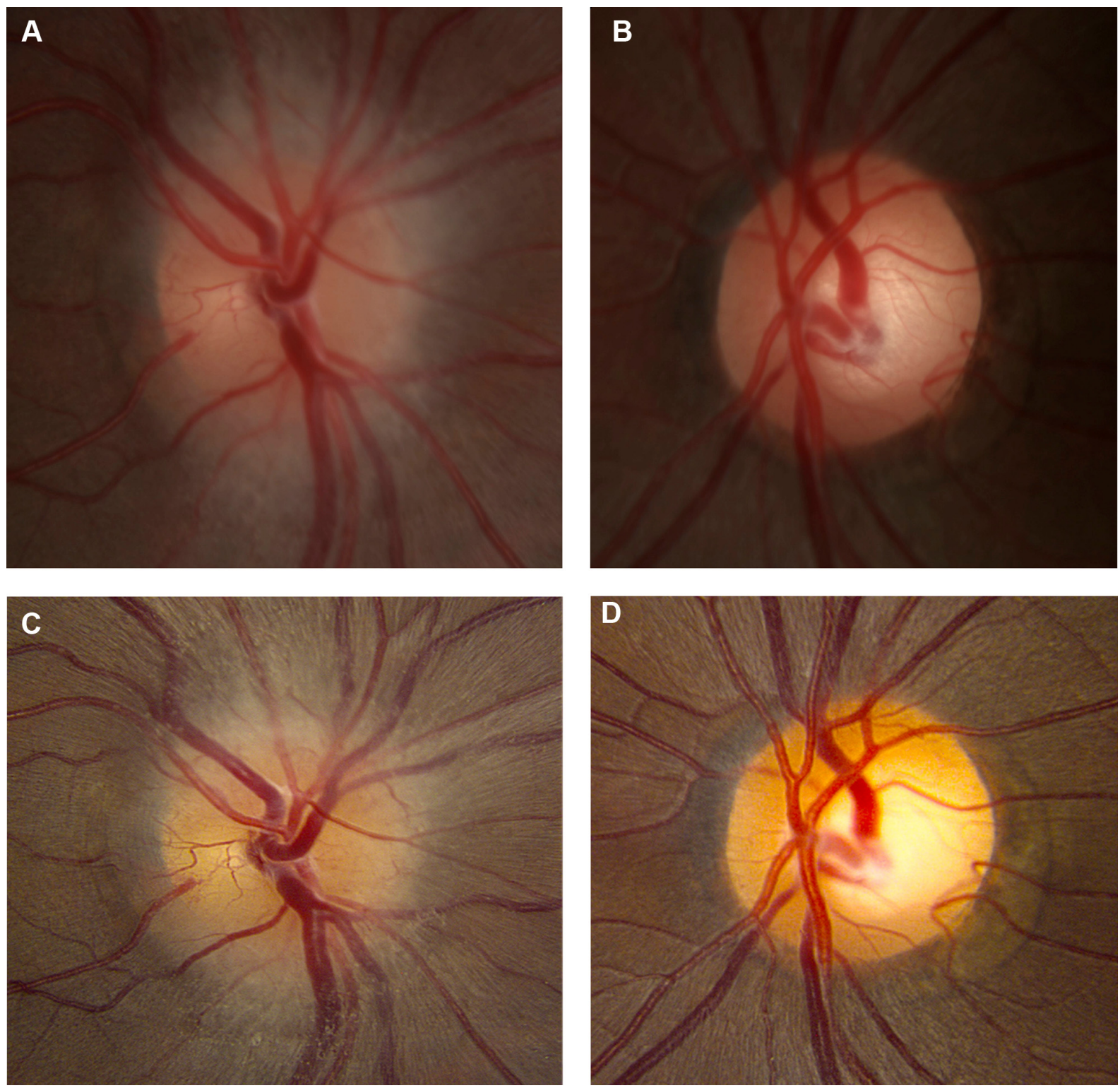

Figure 2 Fundus photos of both eyes.

Notes: (A) At II days after onset of symptoms, the right optic nerve head had diffuse edema, and (B) the left optic nerve head appeared normal. Three weeks after presentation, (C) the right optic nerve head showed persistent edema and (D) the left optic nerve remained normal.

Seven weeks after the onset of symptoms, the vision in the right eye had improved to 20/400; repeat GVF showed expansion of the superior field (Figure 3B), eye pain resolved, and a repeat MRI found decreased right optic nerve enhancement (Figure $1 \mathrm{E}$ and $\mathrm{F}$ ). The patient eventually admitted to intermittent use of several over-the-counter sexual enhancement supplements throughout the course of his steroid treatment without disclosing the exact amount or frequency, which he had discontinued prior to his clinical improvement. Four months after onset of visual loss, his headache and pain with eye movement had resolved. GVF showed improved sensitivity in the superior field and a persistent inferior defect in the right eye (Figure 3C). Visual acuity of the right eye was 20/200, at which point it remained stable.

\section{Discussion}

The association between PDE-5 inhibitors prescribed for erectile dysfunction and optic neuropathy is still under investigation. ${ }^{10,11}$ Recent data demonstrate a small but statistically significant increase in the risk of AION from PDE-5 inhibitor exposure. ${ }^{9}$ While the role of PDE-5 inhibitors in causing ischemic injury to the optic nerve is unknown, there may be an association between AION and PDE-5 inhibitors due to their vasomotor effects on the nitric 


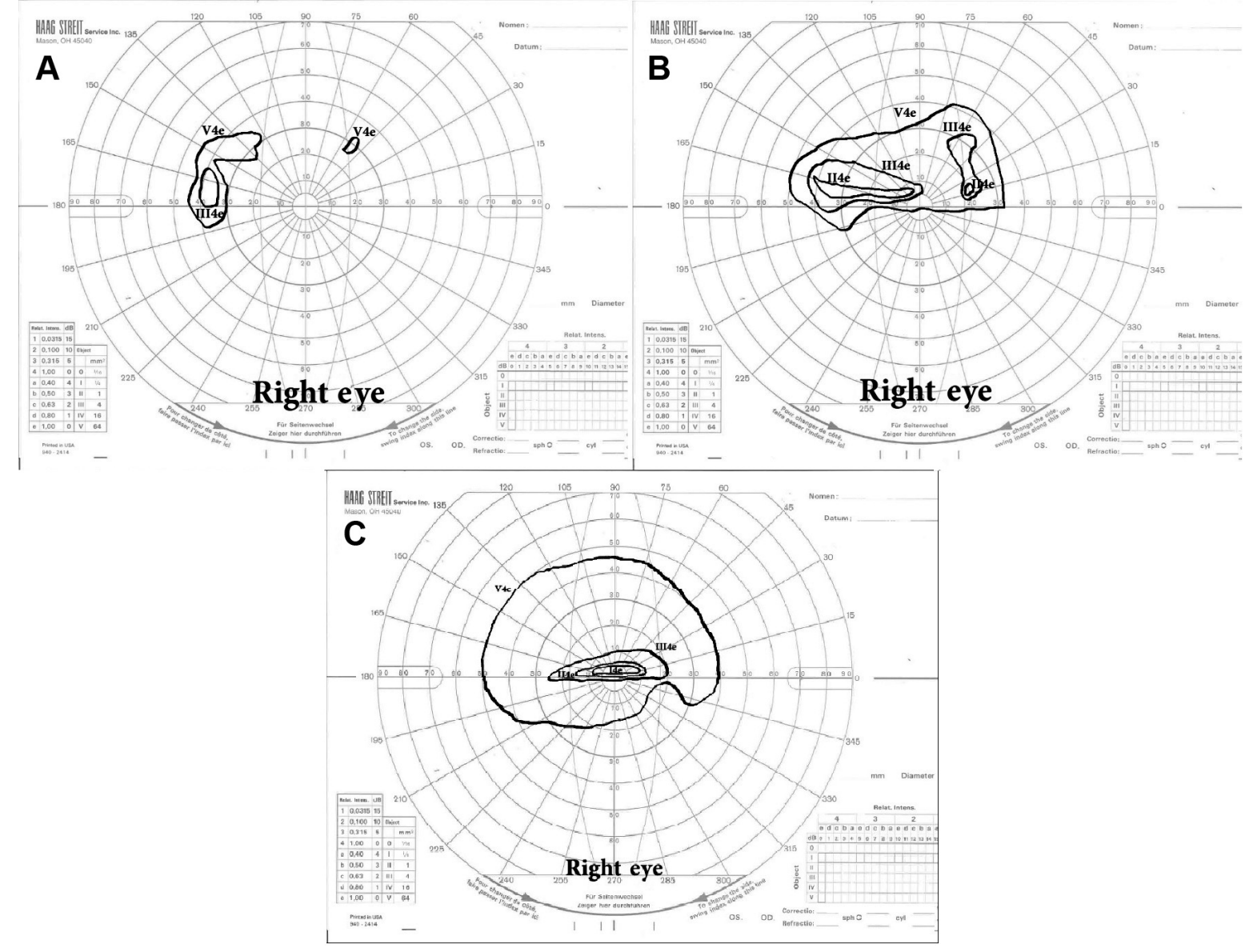

Figure 3 Goldmann visual field of the right eye.

Notes: (A) At 3 weeks after initial presentation, the right eye vision shows small islands in the superotemporal and superonasal fields. (B) At 7 weeks after initial presentation, the right eye shows severe visual field restriction, with small islands of vision remaining superiorly. (C) At 4 months after initial presentation, the right eye vision shows an inferior altitudinal defect.

oxide/cyclic guanosine monophosphate (NO/cGMP) pathway. ${ }^{4}$ The NO/cGMP pathway is important for both regulation of normal ocular blood flow and photoreceptor transduction. ${ }^{3}$ PDE-5 breaks down cGMP and therefore reduces NOinduced vasodilation. ${ }^{3}$ PDE-5 inhibitors increase cGMP concentration and subsequently may lead to vasodilation and reduction in optic nerve perfusion. Optic nerve hypoperfusion may also occur because PDE-5 is found in smooth muscle cells of the systemic arteries and veins throughout the body, and thus PDE-5 inhibitors may induce systemic hypotension because of the mild systemic vasodilator effects. ${ }^{13}$

Our patient developed atypical optic neuropathy and eventually admitted to using several over-the-counter "herbal" sexual enhancement supplements prior to his visual symptoms and throughout his steroid treatment, which had a Chinese label claiming it may contain PDE-5 inhibitors, "male hormones," and "substances that enhance blood production." He did not disclose the amount or frequency of supplement use. The patient's progressive clinical course was not typical of optic neuritis or AION. The patient's age, pain with ocular motility, optic nerve head edema, and optic nerve enhancement on MRI were more consistent with optic neuritis and perineuritis. However, the lack of improvement and progression of the optic nerve enhancement on MRI despite high-dose corticosteroids is not typical for optic neuritis. The MRI findings are also not consistent with AION wherein optic nerve enhancement is not usually present. ${ }^{14-16}$

We hypothesize that the continued use of over-thecounter sexual enhancement supplements may have led to a reduction of optic nerve perfusion, leading to serial ischemic insults. While one case cannot establish association, our case may suggest the potential dangers of unregulated over-the-counter sexual enhancement supplements, and patients using these supplements should be educated about the potential risks. In the event of optic neuropathy, these medications should likely be discontinued to reduce the possible risk of further visual loss to both the affected and the unaffected eyes. Our case also underscores the importance 
of obtaining a careful history of supplements in patients with optic neuropathies.

\section{Acknowledgments}

This work was supported by the National Institutes of Health Center Core (grant P30EY014801), and a Research to Prevent Blindness Unrestricted Grant.

\section{Disclosure}

The authors report no conflicts of interest in this work.

\section{References}

1. Gorkin L, Hvidsten K, Sobel RE, Siegel R. Sildenafil citrate use and the incidence of nonarteritic anterior ischemic optic neuropathy. Int J Clin Pract. 2006;60:500-503.

2. Hayreh SS. Non-arteritic anterior ischaemic optic neuropathy and phosphodiesterase-5 inhibitors. Br J Ophthalmol. 2008;92(12): $1577-1580$.

3. Laties AM. Vision disorders and phosphodiesterase type 5 inhibitors: a review of the evidence to date. Drug Safety. 2009;32(1):1-18.

4. Pomeranz HD, Smith KH, Hart WM, Egan RA. Sildenafil-associated nonarteritic anterior ischemic optic neuropathy. Ophthalmology. 2002;109: 584-587.

5. Azzouni F, Abu Samra K. Are phosphodiesterase type 5 inhibitors associated with vision-threatening adverse events? A critical analysis and review of the literature. J Sex Med. 2011;8:2894-2903.

6. Cullen JF, Chung HW. Mistaken diagnosis of optic neuritis and the possible role of phosphodiesterase-5 inhibitors (sildenafil/Viagra). Med $J$ Malaysia. 2010;65(4):317-318.

7. Su DHW, Ang PS, Tow SLC. Bilateral posterior ischemic optic neuropathy associated with use of sildenafil. J Neuroophthalmol. 2008; 28(1):75.
8. McCulley TJ, Lam BL, Marmor MF, Hoffman KB, Luu JK, Feuer WJ. Acute effects of sildenafil (Viagra) on blue-on-yellow and white-onwhite Humphrey perimetry. J Neuroophthalmol. 2000;20(4):227-228.

9. Pfizer. A study to assess whether PDE5 inhibitors increase the chance of triggering the onset of acute NAION. In: ClinicalTrials.gov [Internet]. Bethesda (MD): National Library of Medicine (US); 2000 [cited May 7, 2014]. Available from: http://clinicaltrials.gov/show/NCT00759174. NLM Identifier: NCT00759174.

10. Bayer. PDE5 inhibitor use and non-arteritic anterior ischemic optic neuropathy (NAION). In: ClinicalTrials.gov [Internet]. Bethesda (MD): National Library of Medicine (US); 2000 [cited May 7, 2014]. Available from: http://clinicaltrials.gov/show/NCT00867815. NLM Identifier NCT00867815.

11. Eli Lilly and Company. A study to determine if there is a possible association between nonarteritic anterior ischemic optic neuropathy (NAION) and phosphodiesterase type 5 (PDE5) inhibitors. In: ClinicalTrials.gov [Internet]. Bethesda (MD): National Library of Medicine (US); 2000 [cited May 7, 2014]. Available from: http://clinicaltrials. gov/show/NCT01131104. NLM Identifier: NCT01131104.

12. Hoitsma E, Faber CG, Drent M, Sharma OP. Neurosarcoidosis: a clinical dilemma. Lancet Neurol. 2004;3(7):397-407.

13. Kloner RA. Cardiovascular effects of the 3 phosphodiesterase- 5 inhibitors approved for the treatment of erectile dysfunction. Circulation. 2004;110(19):3149-3155.

14. Rizzo JF 3rd, Andreoli CM, Rabinov JD. Use of magnetic resonance imaging to differentiate optic neuritis and nonarteritic anterior ischemic optic neuropathy. Ophthalmology. 2002;109:1679-1684.

15. Arnold AC. Ischemic optic neuropathy. In: Miller NR, Newman NJ, Biousse V, Kerrison JB, editors. Walsh \& Hoyt's Clinical NeuroOphthalmology. Vol. I. 6th ed. Philadelphia: Lippincott Williams \& Wilkins; 2005:349-384.

16. Ischemic Optic Neuropathy Decompression Trial Research Group. Characteristics of patients with nonarteritic anterior ischemic optic neuropathy eligible for the Ischemic Optic Neuropathy Decompression Trial. Arch Ophthalmol. 1996;114:1366-1374.
Clinical Ophthalmology

\section{Publish your work in this journal}

Clinical Ophthalmology is an international, peer-reviewed journal covering all subspecialties within ophthalmology. Key topics include: Optometry; Visual science; Pharmacology and drug therapy in eye diseases; Basic Sciences; Primary and Secondary eye care; Patient Safety and Quality of Care Improvements. This journal is indexed on

Submit your manuscript here: http://www.dovepress.com/clinical-ophthalmology-journal

\section{Dovepress}

PubMed Central and CAS, and is the official journal of The Society of Clinical Ophthalmology (SCO). The manuscript management system is completely online and includes a very quick and fair peer-review system, which is all easy to use. Visit http://www.dovepress.com/ testimonials.php to read real quotes from published authors. 